\title{
The Right and Justice Between What is Right and What is Useful
}

\author{
Arjan Vasjari PhD \\ European University of Tirana
}

\section{Doi:10.5901/mjss.2013.v4n6p589}

\section{Abstract}

Is justice useful to the strongest, in other words to the power? Are the right and the usefulness of the power consistent to the justice in its most elaborate principles? Further more, why should the individual be right, in other words, adapt itself to a justice imposed upon him like being useful to the power? These few questions claim to receive much more detailed answers through this article, even though not in an exhaustive manner. First by explaining the concept usefulness and right as being both sides of a good highlighting that these concepts are not opponents and that they can coexist together. The usefulness is more individualistic but it can be developed and transformed into a social usefulness, national useful, universal useful, and it can coexist in a more natural and organic way with the concept right, which in itself is more collective, more inter-subjective. To Epicurus, the right consists in the useful with the mutual relations. The right needs to express something which is useful to the society. A confrontation with John Rawls' Theory of Justice, despite the difficulties it contains, tends to explain that the collective usefulness is right. Right is precisely this, a link between these two concepts.

Keywords: justice, useful, just law, inter-subjectivity, social rule, collective usefulness

\section{Premessa}

Nella famosa opera di Platone la "Repubblica",c'è un acesissimo dibatito tra Socrate e Trasimaco circa il modo di concepire la giustizia. "La giustizia - dichiara Trasimaco - è l'utile del più forte". Secondo il sofista "... ciascun governo istituisce leggi per il proprio utile; la democrazia fa leggi democratiche, la tirannide tiranniche e allo stesso modo gli altri governi. E una volta che hanno fatto le leggi, proclamano che il giusto per i governati è ciò che è invece il loro proprio utile, e chi se ne allontana lo puniscono come trasgressore della legge ed ingiusto. Questo, è quello che dico giusto, il medesimo in tutte quante le poleis, l'utile del potere costituito. Ma, se non erro, questo potere detiene la forza. Così ne viene, per chi sappia ben ragionare, che in ogni caso il giusto è sempre identico all'utile del più forte (Repubblica,Platone,pg 338-339).

Per Trasimaco la giustizia si riduce in tutti i casi a uno strumento del potere costituito - sia esso democratico, aristocratico o tirannico - finalizzato al suo utile.

Per Socrate il giusto puo essere utile ma questa non puo essere,neccessariamente "del più forte". Trasimaco precisa che per "più forte" intende colui che è più competente e non sbaglia: il governante, in quanto è al governo e fin tanto che riesce a rimanerci, non sbaglia, e stabilisce il giusto come suo utile(ivi pg 341).

\section{II giusto e l'utile del potere costituito}

Praticamente,questo coincide,grosso modo, con il nucleo essenziale del positivismo giuridico: il giusto è obbedire alle leggi imposte da chi dettiene il potere.Quindi,la giustizia si identifica con l'utile dei governati che venne imposto dai governanti secondo il loro concetto sul giusto. Non c'è dubbio che siamo di fronte alla visione meramente politica della giustizia che puo essere tradota cosi: a-governnanti sono i piu forti,b-governanti fanno leggi nel loro interesse;cgovernanti sanno quall'è il loro miglior interesse.

$\mathrm{Ma}$,è vero che il giusto e l'utile del potere costituito coincidono con la giustizia,nelle sue piu elaborate concezzioni? E poi,perché il singolo dovrebbe essere giusto, cioè,perché dovrebbe adeguarsi a un diritto imposto nell'utile del potere costituito?|l giusto e l'utile rappresentano i due lati piu importanti di un bene,il quale,per realizzarsi necessita la volonta dell'individuo sia nella sua dimensione individuale sia in quella colletiva. Va detto subito: il giusto e l'utile non sono due cose opposte. 
L'utile,che si impone all'uomo come per necessita dell'istinto,significa per esso un complesso di esigenze che li assicurano la sua conservazione.Partono da questa concezzione autori come Hobbes,Bentham,Spencer ecc,i quali pongono l'utile come la base del diritto. Secondo loro,l'obbietivo principale della legge è quello di assicurare la conservazione e la difesa dell'individuo, quindi il precetto sostanziale del diritto è quello di neminem laedere.Come si vede,cosi esposto,il concetto dell'utile,è piu propenso ad essere legato con l'essere individuale della persona umana.

Dall'altro canto,il giusto,corrisponde di preferenza alla natura associativa dell'individuo,cioe al suo essere colletivo. Questo vuol dire che l'individuo puo essere costretto anche con la forza per la sua realizzazione perche,senza l'osservanza del giusto non sarebbe possibile la coesistenza pacifica sociale.In altre parole,il giusto non ha a che fare con l'istinto individuale dell'uomo ma si fonda invece,sulla socialita. II giusto,si impone si' come un mezzo di conservazione ma anche come un mezzo di perfezzionamento. Dunque,il giusto "obbliga" l'individuo di uscire dalla sua natura circostante e lo costringe a instaurare un rapporto con l'altro.Facendo cosi,da una parte lui asserisce il suo diritto ad avere tutto cio che gli appartiene,dall'altra si sottomette all'obbligo di attribuire a ciascuno il suo.

Ovviamente che questa tesi non riguarda i contenuti della giustizia, ma solo la sua funzione, nella prospettiva di un soggetto morale.Chi vuol essere giusto anche solo parzialmente, si fa asservire dal potere costituito.

Ritornando dall'utile,ossia l'interesse individualistico,quando domina l'uomo,si verrebe in certo modo a fraporre tra uomo e uomo,trasformando il rapporto da homo homini deus in homo homini lupus,combaciandosi perfettamente con la tesi di Hobbes della guerra di ciascuno contro tutti. Questo perche,individuo,isolatamente parlando,essendo esclusivamente fino a se stesso e non vedendo oltre,puo conservare la sua esistenza soltanto battendo il prossimo,uguale come nella societa primitiva.E' l'unico modo per realizzare il suo utile. Ma l'utile,non e' soltanto un bisogno egositico della persona perche puo essere anche un'utile sociale,un'utile nazionale,un'utile universale. Svilupandosi cosi,utile combacia col giusto e in questo modo l'utile si trasforma in uno stimolo efficace alla convivenza sociale. In questa tipologia dell'utile Kant scopre la solidarieta degli interessi commerciali delle nazioni come garanzia di pace universale (Immanuel Kant, Per la pace perpetua, 1795, VI capitolo).Dice Romagnosi "...è dimostrato che l'utilita forma un carattere perpetuo ed essenziale di qualunque diritto o dovere e che questa utilita, è l'utilita regolata,non l'utilita sregolata,che in sostanza coincide con il giusto bene inteso..." (Giandomenico Romagnosi,Diritto naturale, ed.Guasti, Prato,pg 91). L'utile costituisce la materia del giusto e finche non c'è l'utile,non comincia il giusto.

\section{3. "L'utile" sociale e "il giusto" colletivo}

Riferendosi al Epicuro nei suoi Scritti Morali, il diritto,nella sua nozione generale,è medesimo per tutti. Esso consiste nell'utile da osservarsi nei rapporti comuni. Ma se guarda a ciò che ciascuna regione ha di specifico,allora, non per tutti il diritto sia il medesimo. Molto saggiamente il filosofo della Grecia antica continua: "Delle cose ritenute giuste quella che l'esperienza attesti essere utile nei bisogni dei rapporti comuni, ha quanto occorre per essere base di diritto, sia essa per tutti la medesima o non sia. Ma se una cosa sia stata solo posta per legge, ma non si dimostri conforme all'utile dei rapporti comuni, essa non ha più la natura del giusto. $E$ se anche l'utile, che è a fondamento del diritto, si muti, ma per un certo tempo sia conforme alla nozione che un popolo ha di esso, non per questo per tutto quel tempo esso non è stato giusto, ove non ci si confonda la mente con parole vuote di senso, ma si guardi alle cose".(Epicuro,Scritti Morali,pg.56,Milano 1987).E poi, premendo sulla mutabilita del rapporto giusto-utile o meglio dire sulla dipendeza di questo rapporto dal contesto,esso continua:" Dove, immutate restando le condizioni circostanti, norme ritenute giuste si rivelino alla prova dei fatti non conformi alla nozione del diritto, tali norme non erano giuste. Dove, invece, la condizione delle cose essendo mutata, norme poste come giuste non risultano più utili, ivi s'ha da dire che esse furono giuste fino a che furono utili ai reciproci rapporti dei singoli facenti parte della comunità politica, ma che in seguito non furono giuste quando non furono più utili".

Quello che (ri)scopriamo nelle parole epicuriane riguarda la mutabilita permanente della giustizia e del diritto. La giustizia non è, e non può essere eternamente definita perchè, essa è obbligata a seguire i passi della societa, il cambiamento dei bisogni dell'individuo, volti costantemente a migliorare la sua condizione sociale, materiale e spirituale. Perche cambia il concetto di utile, cambia il concetto di equita e questo, obbligatoriamente, vuol dire che deve aggiustarsi anche la giustizia. In altre parole, la giustizia deve essere in evoluzione permanente in modo tale da potersi tener fede sempre ai principi di ugualianza, in base al quale tutti gli uomini sono uguali e il principio di differenza e unicita che caratterizza ognuno dei individui in quanto quanto singoli e speciali. Quello che invece non cambia è il concetto che, il giusto è cio che giova alla colletività e siccome quest'ultima si costruisce intorno al patto sociale allora,giustizia e il senso del giusto derivano dal medesimo patto. 
E' indiscutibile che esiste un rapporto intrinseco tra il giusto ed il diritto.D'altro canto,esistono norme ritenute giuste,ma che alla prova dei fatti non coincidono con il concetto diritto.Perche questo? Perche il giusto non è un concetto,il cui carattere sia del tutto soggettivo ed individuale,come quello dell'utile. II concetto del giusto l'individuo lo riscopre quando costruisce una relazione con altro e per cio,il giusto rappresenta "l'idea architettonica della societa umana". (Buckle, Henry Thomas, History of civilization in England, 1857.pg.200). Questo vuol dire che il giusto sia un concetto,sostanzialmente,sociale. "Il giusto per dirlo con Platone, è scritto a picccole lettere nell'individuo e a grandi lettere nella societa umana". (Platone, La Repubblica,libro II). Ovunque, il giusto,impedisce il crash degli interesi particolari orientando l'uomo a essere partecipe nella vita sociale. Da qui si puo desumere con certezza che il diritto,prima di essere norma è relazione sociale. Questa non è una novita perche cosi è stato da sempre. "E' ormai evidente che il diritto dei popoli primitivi non è un elenco delle cose da fare o da non fare,ma un programa di vita comune"(Lon Fuller,Human Interaction and Law,in The Principles of Social Order,1981,pg.212).Di conseguenza,il diritto dovrebbe esprimere qualcosa che sia utile alla societa ma nello stesso tempo possiamo dire che la giustizia si fa solo in rapporto con gli altri,cioe,dentro la societa. E partendo da questo concetto che si fa ad essere giusti.

Nel giusto ocorre in modo permanente un elemento mutabile,transitorio che coincide con l'utile e un elemento costante che corrisponde al concetto dell'equo,tipico del diritto. Sono due principi,apparentemente opposti che s'incontrano nel dominio del giusto, dando vita a quei dualismi che sono alla base della giurisprudenza dei romani. Basti ricordare i dualismi della giustizia commutativa e della giustizia distributiva;quelli di ius strictum e del equitas,del ius commune e del ius singulare, della lettera e dello spirito della legge,d ella forza e della ragione. Tutti questi dualismi,che sono alla base del diritto, sono eterni e costanti nello svolgimento delle legislazioni delle societa. Quindi,la giustizia giusta,è quella che si rispechia nella sua duplice dimensione: di utilita e di equita. Utilita ed equita che garantiscono la coesione del corpo sociale,la sua conservazione,la convivenza colletiva.

Esiste,puo esistere una gerarchia,un subordinazione fra utile e giusto?Sicuramente si. Chi crede che questi due concetti cosi cari per il diritto,siano fra di loro in una condizione di parita assoluta,sbagliano in modo grossolano. Se cosi fosse,allora le legislazioni sarebbero immutevoli. Ma nella storia evoluzionistica delle legislazioni si manifesta un processo uniforme e costante per cui,esse dapprima si ispirano di preferenza all'utile e alla necessita della conservazione e difesa ma col passare del tempo cercano di essere piu giuste. In linea con questo possiamo aggiungere anche un altra cosa: riconoscendo che il diritto ed il concetto del giusto che vi corrisponde abbiano una funzione propria nella societa,distinta da quella dell'utile,si puo ribadire che l'individuo e la societa,possono cercare e pretendere di realizare il proprio utile,entro i perimetri del diritto e del giusto. Per escludere ogni malinteso va sottolineato:nel continuo attrito ed intreccio tra il giusto e l'utile si verifica una prevalenza del giusto sul utile.Ma,giusto e utile non si escludono a vicenda,anzi. II caso dei cosidetti hard cases è tipico per spiegarci meglio tutto questo. Un caso difficile è un caso che richiede la revisione dei criteri usuali per la sua soluzione. Praticamente,la soluzione sara quella migliore nei casi concreti. Per il diritto che tende di realizzare il giusto,la soluzione migliore sara quella piu giusta il quale non sara tale se non sara la piu utile. Quindi,non si puo definire utilitarismo cinico se si dichiara che utile ci aiuta a dividere il diritto giusto da quello ingiusto.

\section{Massimizzazione distributiva delle diseguaglianze}

E' di significativo interesse se in questo ambito di vedute,costruiamo un confronto(anche se non esauriente) con la teoria della giustiza come equita di John Rawls.Quello che c'i interessa è il cosidetto principio di differenza,un principio che ha come scopo quello di regolare la distribuzione giusta di beni,una volta garantita l'ascrizione delle liberta fondamentali uguali per ciascuno secondo il primo principio. Secondo Rawls,il principio di equita distributiva mira a rendere uguale la disegualianza delle liberta. Ma,il principio di differenza altro non è che un variante del principio distributivo. Scrive Rawls: "Tutti i valori sociali-liberta e opportunita,ricchezza e reddito,e le basi del rispetto di sè-devono essere distribuiti in modo eguale,a meno che una distribuzione ineguale di uno 0 di tutti questi valori,non vada a vantaggio di ciascuno.Quindi,'ingiustizia,coincide semplicemente con le inegualianze che non vanno a beneficio di tutti" (John Rawls, Theori of justice,1982,pg.65). Puo sembrare che la posizione di Rawls preme sul giusto escludendo l'utile.Ma cosi non è. $E$ ' vero che,la distribuzione delle disegualianze è un applicazione del principio del giusto. Una societa costruita sulla "disegualianza delle disegualianze" sarebbe stato una societa ingiusta. Quindi spalmando le disegualianze significa ristabilire il giusto. Ma questo non è e non puo essere un operazione che tende a rimettere in ordine una giustizia violata. In altre parole,questa operazione non si fa unicamente perche cosi è piu giusto ma perche,facendo cosi, è anche piu utile per la convivenza pacifica della societa. Riducendo soltanto come una necessita di ristabilire il giusto in sè escludendo il principio dell'utile,si realizza la giustizia formale ma non quella sostanziale. In altre parole,distribuendo le 
diseguaglianze si realizza un interesse personale giusto ma non un interesse personale isolato ed esclusivo.Distribuendo le diseguaglianze viene realizzato sicuramente un interesse personale ma,operando cosi,si incrementa anche l'utile colletivo e di conseguenza si salvaguarda l'interesse generale. Distribuendo le diseguaglianze non si fa altro che,si include l'equità nel principio utilitarista della massimizzazione dell'utile collettivo, per cui non si parlerebbe più di massimizzazione aggregativa dell'utilità, ma di massimizzazione distributiva delle diseguaglianze.

Lo stesso ragionamento vale anche per la tesi centrale dell'opera di Rawls,quella del arbitrarietà delle dotazioni iniziali,naturali e sociali di un individuo. L'egualianza delle opportunita a scapito della posizione originaria (lotteria naturale lo chiama Rawls),nella sua essenza,non riguarda la correzione morale delle disegualianze a causa delle differenze sociali o economiche. Se cosi fosse questa sarebbe semplicemente una giustificazione etica e basta,un opera di moralizzazione. Egualianza delle oportunita è una condizione per la coestistenza pacifica degli uomini,cioè è utile per l'ordine sociale. Riequilibrare le differenze attraverso le pari opportunita,facciamo una cosa giusta sicuramente ma,sopratutto facciamo una cosa utile per la salute del corpo sociale.Senza dimenticare che in una società libera, $\mathrm{i}$ talenti e le doti delle persone avvantaggiano davvero gli altri, e non solo se stessi,quindi essendo una risorsa collettiva sono utili a tutti. In questo senso,la teoria della giustizia di Rawls,vacilla nelle sue basi perche bon prende in considerazione un simile fatto. Cosa puo essere dietro questo? E' molto difficile trovare una risposta. C'i limiteremo a portare qui quella del suo "avversario",Nozick: "'È così implausibile affermare che alla base di questa concezione della giustizia, a formare parte della sua idea fondamentale, c'è l'invidia?"( Nozick, R., Anarchia, Stato e utopia, traduzione italiana di G. Ferranti, Milano, il Saggiatore, 2000, p. 238)

La pratica giuridica è un altro elemento che ci' aiuta a trovare un punto d'incontro tra il giusto e l'utile. La domanda che ci poniamo a riguardo puo essere questa: qual'è la maniera migliore di praticare il diritto? La risposta puo essere: quella piu giusta. D'istinto ci vienne un altra domanda: qual'è quella giusta? Quella giusta è la pratica giuridica che si pratica giustamente. Ma è sufficente che si pratica giustamente,cioè secondo i canoni del giusto che possono variare? Non è sufficente finche la pratica giuridica non ha come suo fine quello di perseguire la giustizia.Sappiamo bene che la giustizia è questione di grado. Questo vuol dire che la pratica giuridica puo essere piu o meno giusta o piu o meno ingiusta. Quando è giusta è anche utile perche combacia con l'interesse colletivo il quale si puo realizzarsi soltanto "all'interno di un livello insuperabile di guardia costituito dall'eguaglianza e dall'equita" (Francesco Viola,Diritto vero e diritto giusto,in "Persona y derecho,24,1991,pg 255). Praticamente,questa consapevolezza parte dall'idea dell'utile colletivo come il mecanismo par exellence che puo garantire una pratica giuridica giusta ma non in astratto. L'utile riesce a coniugare il giusto della pratica giuridica con l'interese colletivo,garantendo razionalmente una soddisfacente convivenza civile tra i consociati.

\section{Conclusione}

Ritornando sul dibatito iniziale tra Socrate e Trasimaco,possiamo dire con certezza che la giustizia non è l'utile del piu forte. Piutosto è l'utile della coesistenza giusta e pacifica tra gli individui della societa. Sia se si tratta di giusto legale (cioè quando è conforme ad una norma giuridica) sia se si tratta di giusto morale (cioè quando è conforme ad una norma morale) senza escludere nessuna delle due. La conservazione e la convivenza dentro il corpo sociale rappresentano l'utile ed il giusto allo stesso tempo. Questa conclusione ha senso se il diritto vienne concepito sopratutto come pratica sociale. Sono le azioni comuni e le relazioni intersoggettive con i rispettivi valori e interessi di importanza colletiva che fanno il diritto un anello di congiuzione tra il giusto e utile. II giusto e l'utile del potere costituito possono coincidere con la giustizia ed il diritto ma il diritto e la giustizia possono essere giusto ed utile se neccessariament coincidono con l'esistenza della specie umana in una dimensione colletiva.

\section{Bibliografia}

Platone,La Repubblica,pg 338-339, ivi pg 341

Immanuel Kant,Per la pace perpetua,1795,VI capitolo

Giandomenico Romagnosi,Diritto naturale,ed.Guasti,Prato,pg 91

Epicuro,Scritti Morali,pg.56,Milano 1987

Henry Thomas Buckle,History of civilization in England,1857.pg.200

Platone,La Repubblica, libro II

Lon Fuller,Human Interaction and Law,in The Principles of Social Order,1981,pg.212

John Rawls, Theori of justice,1982,pg.65

Nozick R. Anarchia,Stato e utopia,Milano ,il Saggiatore,p.238

Francesco Viola ,Diritto vero e diritto giusto,in "Persona y derecho,24,1991,pg 255 